\title{
Acervos digitais e o Uso das Tecnologias de Informação e Comunicação em Arquivos Brasileiros
}

\section{Acervos Digitales y el Uso de las Tecnologías de la Información y la Comunicación en Archivos Brasileños}

\section{Digital Collections and the Use of Information and Communication Technologies in Brazilian Archives}

\author{
Luciana Piazzon Barbosa Lima' \\ Isabela Bertolini Coelho"
}

\section{Palavras-chave: \\ Tecnologias de Informação e Comunicação (TIC) \\ Arquivos}

Acervos Digitais

\section{Resumo:}

Os arquivos podem contribuir para a ampliação do acesso à cultura por meio do uso das tecnologias da informação e comunicação (TIC), sobretudo pela oferta de bens culturais na Internet. A pesquisa TIC Cultura, realizada pelo Comitê Gestor da Internet no Brasil (CGI.br) por meio do Centro Regional de Estudos para o Desenvolvimento da Sociedade da Informação (Cetic.br), do Núcleo de Informação e Coordenação do Ponto BR (NIC.br), analisa a infraestrutura e os usos dessas tecnologias por equipamentos culturais brasileiros. $\mathrm{O}$ artigo apresenta os resultados do estudo para o caso específico dos arquivos, com foco, em especial, nos indicadores sobre a apropriação das TIC para a criação de acervos digitais e sua disponibilização na Internet. Os dados revelam ser este um potencial ainda pouco explorado por estas instituições, que poderia ser mais bem aproveitado com o desenvolvimento de políticas públicas para investimento e capacitação para a digitalização de acervos. 


\section{Resumen:}

Los archivos pueden contribuir a la ampliación del acceso a la cultura a través del uso de las tecnologías de la información y la comunicación (TIC), sobre todo por la oferta de bienes culturales en Internet. La investigación TIC Cultura, realizada por el Comité Gestor de Internet en Brasil (CGI.br) por medio del Centro Regional de Estudios para el Desarrollo de la Sociedad de la Información (Cetic.br), del Núcleo de Información y Coordinación del Punto BR (NIC.br), analiza la infraestructura y los usos de esas tecnologías por equipamientos culturales brasileños. El artículo presenta los resultados del estudio para el caso específico de los archivos, con foco, en particular, en los indicadores sobre la apropiación de las TIC para creación de acervos digitales y puesta a disposición en Internet. Los datos revelan que este es un potencial aún poco explotado por estas instituciones, que podría mejor aprovecharse con el desarrollo de políticas públicas para la inversión y la capacitación para la digitalización de acervos.
Palabras clave:

Tecnologías de la Información y la Comunicación (TIC)

Archivos

Digitalización de Acervos

\section{Keywords:}

Information

and Communication

Technologies (ICT)

Archives

Digital Collections

\section{Abstract:}

Archives can contribute for expanding access to culture through the use of information and communication technologies, mainly through the provision of cultural goods on the Internet. The ICT in Culture survey, developed by the Brazilian Internet Steering Committee (CGI. br) through the Regional Center for Studies on the Development of the Information Society (Cetic.br),a department of the Brazilian Network Information Center (NIC.br), analyses the infrastructure and use of these technologies by Brazilian cultural facilities. The article presents the results of the study for the specific case of Brazilian archives, focusing, in particular, on the indicators on the appropriation of ICT for creating digital collections and making them availableon the Internet. The data reveal that this potential is not fully harnessed by these institutions and could be better explored with the development of investment and capacity-building public policies for the digitalization of collections. 


\section{Acervos digitais e o Uso das Tecnologias de Informação e Comunicação em Arquivos Brasileiros}

\section{Introdução}

A disseminação das tecnologias da informação de informação e comunicação (TIC) em diversos setores têm gerado impactos não só de ordem econômica, como também simbólica. No campo da cultura, as TIC estão sendo incorporadas nas práticas culturais dos cidadãos e na forma de atuação de arquivos, bibliotecas, museus, etc., gerando mudanças profundas na maneira como criamos, preservamos e difundimos acervos e bens culturais. Nesse sentido, medir a produção e a fruição desses bens mediadas pelas TIC é de alta relevância para a formulação e implantação de políticas públicas.

Em âmbito internacional, o Plano de Ação da Cúpula Mundial da Sociedade da Informação (World Summit on the Information Society - WSIS) trazia, dentre os objetivos a serem alcançados até 2015, o de conectar todas as bibliotecas públicas, museus, postos de correios e arquivos nacionais com as TIC - objetivo monitorado pela Partnership on Measurement ICT for Development por meio de indicadores sobre o acesso à Internet, a presença na Web e a digitalização e disponibilização de itens por esses equipamentos (UNIÃO INTERNACIONAL DE TELECOMUNICAÇÕES [UIT], 2015).

Nessa mesma direção, entre as recomendações da Organização das $\mathrm{Na}$ ções Unidas para a Educação, a Ciência e a Cultura (Unesco) para o desenvolvimento de sociedades do conhecimento, a ampliação de conteúdos disponíveis para acesso universal destaca o papel a ser desempenhado pelos principais centros de conhecimento - tais como instituições de Ensino Superior, centros de pesquisa, mu- seus e bibliotecas - na produção e disseminação do conhecimento através de redes possibilitadas por conexões de baixo custo e alta velocidade (UNESCO, 2005).

A Unesco também tem discutido, mais recentemente, diretrizes operacionais para implementação da Convenção sobre a Proteção e Promoção da Diversidade de Expressões Culturais no ambiente digital (UNESCO, 2017). Além do princípio de universalidade da Internet, é recomendada a criação de políticas para ampliar as competências digitais do setor, contemplando a criação, produção, disseminação e fruição de bens e serviços culturais e incluindo o papel das instituições da área na oferta de acesso on-line à diversidade de expressões culturais - o que demanda, por sua vez, o provimento de ferramentas digitais para estas instituições.

Assim, os estabelecimentos que desempenham atividades culturais têm a oportunidade de ampliar seu campo de atuação e de diálogo com o público a partir da adoção intensiva e estratégica das TIC, por meio da presença na Internet e da oferta de bens e serviços on-line. Tratando especificamente de museus e instituições arquivísticas, um estudo da Comissão Europeia (EUROPEAN COMISSION, 2002, p. 12) aponta ainda que a inovação tecnológica tem grande importância para as estratégias de valorização e difusão de acervos: "As tecnologias de informação e comunicação desempenharão um papel fundamental para criar e distribuir esses novos conteúdos, que vão muito além do estágio atual de oferecer acesso a informações sobre os objetos do patrimônio cultural."

A mensuração do uso dessas tecnologias por equipamentos culturais apresenta assim um potencial estratégico para formulação e implantação de iniciativas no setor. No Brasil, dados sobre TIC e cultura podem ainda contribuir no monitoramento de metas presentes no Plano Nacional de Cultura (PNC), formulado pelo Ministério da Cultura 
(Minc) com a participação da sociedade civil e aprovado em 2010. Nesse sentido, a meta 40 do PNC propõe a disponibilização na Internet de conteúdos que estejam em domínio público ou licenciados e a meta 41 indica que $100 \%$ das bibliotecas públicas e $70 \%$ dos museus e arquivos disponibilizem informações sobre seu acervo no Sistema Nacional de Informações e Indicadores Culturais (SNIIC) (MINC, 2012).

\section{A Pesquisa TIC Cultura: objetivos e aspectos metodológicos}

Para permitir o acompanhamento da implementação dessas iniciativas no Brasil, o Comitê Gestor da Internet no Brasil (CGI. br), por meio do Centro Regional de Estudos para o Desenvolvimento da Sociedade da Informação (Cetic.br), departamento do Núcleo de Informação e Coordenação do Ponto BR (NIC.br), deu início à Pesquisa sobre o Uso das Tecnologias de Informação e Comunicação nos Equipamentos Culturais Brasileiros -TIC Cultura. Baseada em definições metodológicas reconhecidas para a medição do acesso e uso das TIC para produção de dados comparáveis internacionalmente, a pesquisa tem como objetivos gerais a investigação da influência das TIC nas práticas culturais da população e o levantamento de informações sobre acesso e uso dessas tecnologias por equipamentos culturais brasileiros. Precedida por um estudo qualitativo sobre práticas culturais de indivíduos de diferentes grupos sociais (CGI. br, 2017a), a pesquisa TIC Cultura 2016, objeto deste artigo, é a primeira edição do estudo quantitativo realizado junto a equipamentos culturais (CGI.br, 2017b).

Assim, a pesquisa TIC Cultura 2016traz insumos para a compreensão do cenário de infraestrutura, uso e gestão das TIC nos equipamentos culturais brasileiros, incluindo arquivos, bens tombados, bibliotecas, cinemas, museus, pontos de cultura e teatros. São objetivos específi- cos do estudo mapear a infraestrutura de TIC em equipamentos culturais públicos e privados e investigar os usos das tecnologias nessas instituições, com foco na gestão interna, no contato com os públicos e na formação e digitalização de acervos.

Ainda que as políticas culturais se estruturem de modo mais abrangente, os equipamentos culturais são um importante locus de efetivação do acesso à cultura, tanto pela oferta de atividades de formação e programação cultural, quanto pela preservação e difusão de acervos. Constituem-se, assim, como espaços privilegiados para realização de diferentes etapas do ciclo cultural - da criação e produção à disseminação, exibição e consumo de bens e serviços culturais (UNESCO, 2009).

No contexto brasileiro, dados da Pesquisa de Informações Básicas Municipais (Munic) apontam que, em 2014, 97,1\% dos municípios possuíam ao menos uma biblioteca pública, $27,2 \%$ possuíam museu, $23,4 \%$ teatro ou sala de espetáculo, $21,7 \%$ arquivo público e/ou centro de documentação e 10,4\% cinema (IBGE, 2015). Há, portanto, disparidades importantes quanto à presença dos diferentes tipos de equipamentos culturais espalhados pelo país. Dado que a Internet apresenta-se como um importante meio de ampliação do acesso à cultura, sobretudo em localidades em que os equipamentos culturais não estão presentes, como apontam os resultados do estudo qualitativo conduzido previamente (CGI.br, 2017a), a concentração regional dos equipamentos amplia a importância da disponibilização de bens e serviços culturais on-line.

Em termos metodológicos, a pesquisa TIC Cultura 2016 foi conduzida através de Entrevista Telefônica Assistida por Computador (CATI) a partir de um questionário estruturado aplicado com os principais gestores dos equipamentos, preferencialmente com domínio sobre o funcionamento da instituição tanto no que se refere a aspec- 
tos administrativos quanto à infraestrutura de computador e Internet. A coleta de dados da pesquisa ocorreu entre os meses de novembro de 2016 e abril de 2017.

O recorte estabelecido para este artigo trata especificamente do caso dos arquivos brasileiros. Definidos de acordo com a legislação existente, os arquivos compreendem instituições que têm por finalidades a guarda, a preservação e a disponibilização de "conjuntos de documentos produzidos e recebidos por órgãos públicos, instituições de caráter público e entidades privadas, em decorrência do exercício de atividades específicas, [...], qualquer que seja o suporte da informação ou a natureza dos documentos" (Lei n. 8.159, 1991). A população de referência da pesquisa é definida como os arquivos cadastrados no Conselho Nacional de Arquivos (Conarq), compreendida por 275 arquivos. Devido ao pequeno tamanho do cadastro, foi utilizada a abordagem censitária, ou seja, todos os arquivos cadastrados fizeram parte da pesquisa. No entanto, algumas das instituições não quiseram responder ao questionário, de tal forma que a taxa de resposta obtida foi de $65 \%$.Com isso, o peso básico de cada instituição foi corrigido para não resposta dentro de cada região.

Ao levantar informações sobre a apropriação das TIC pelos arquivos brasileiros, a pesquisa também produziu dados que caracterizam essas instituições em diferentes aspectos. Na seção inicial do artigo, são apresentados a distribuição regional, o porte (definido pelo número de pessoas que atuaram na instituição de forma remunerada ou voluntária nos 12 meses anteriores à pesquisa), a natureza jurídica e as fontes de recursos dos arquivos, bem como os principais indicadores de posse e uso das tecnologias de informação e comunicação, incluindo computador, Internet e telefone celular. Desse modo, é possível verificar que o grau de institucionalidade e o escopo de atuação das instituições se refletem na infraestrutura de TIC disponível.
A partir disso, o artigo apresenta os resultados acerca do uso das TIC pelos arquivos brasileiros com foco nos indicadores acerca dos acervos das instituições, que incluem os tipos de acervos formados, o nível de digitalização e as formas de disponibilização dos mesmos, assim como dificuldades encontradas no processo de digitalização'II . Com isso, o recorte estabelecido privilegia uma análise sobre 0 uso dessas tecnologias para preservação e difusão de bens culturais que fazem parte do acervo dessas instituições. Por fim, conclui com alguns indicativos para uma agenda de políticas públicas que leve em conta as potencialidades e desafios para o uso das TIC nos arquivos brasileiros.

\section{Perfil institucional e infraestrutura de TIC nos arquivos brasileiros}

Os arquivos brasileiros, de acordo com os dados da TIC Cultura, eram instituições de natureza majoritariamente pública (80\%), tanto de nível municipal (32\%), quanto estadual (23\%) e federal (25\%). Em 2016, cerca de metade dos arquivos estava instalada em prédios públicos (52\%), além de uma proporção relevante deles utilizar propriedade privada das instituições (18\%) e espaços alugados (15\%). Outro aspecto que reforça o caráter público da maior parte dos arquivos do país é o indicador de fonte de recursos: $75 \%$ deles afirmaram ter como principal fonte os órgãos governamentais - sendo $30 \%$ municipais, $21 \%$ estaduais e $24 \%$ federais.

Quanto ao tamanho e perfil de suas equipes, a pesquisa mostra que a maioria dos arquivos não possuía voluntários e que a maior parte deles era de pequeno e médio portes, possuindo entre 1 e 50 pessoas que trabalharam de forma remunerada (Tabela 1). Para além da preservação e difusão de seus acervos, as atividades dos arquivos eram principalmente a realização de exposições $(70 \%)$ e de oficinas ou formação para 
o público (57\%), o que condiz com seu caráter de instituições custodiais de memória.

Com relação à infraestrutura de TIC, em 2016 os arquivos apresentaram as maiores proporções de posse (97\%) e uso de computador ( $99 \%$ ) entre todos os tipos de equipamentos culturais pesquisados. Os arquivos foram também o tipo de equipamento cultural que apresentou os maiores percentuais de instituições com um número maior de computadores (40\% tinham 11 ou mais computadores) quando comparado aos demais tipos de equipamentos investigados. O telefone celular, por outro lado, foi usado para fins de trabalho em apenas metade dos arquivos $(52 \%)$.
O mesmo ocorre com uso de Internet (97\%), com resultados que revelaram a universalização dessas tecnologias nesse tipo de instituição. Ainda assim, a posse de WiFi não estava plenamente difundida (62\%), sendo a disponibilização da conexão para o público ainda bastante incipiente $(41 \%)$.

No que se refere aos tipos de conexão à Internet, os mais utilizados pelos arquivos foram as conexões via fibra ótica (45\%), a cabo (41\%) e DSL (40\%). A faixa de velocidade para download mais citada foi de $5 \mathrm{Mbps}$ a $10 \mathrm{Mbps}(14 \%)$, sendo o percentual relevante, em comparação com os outros tipos de equipamentos e também com os arquivos que disseram contratar velocidade acima de $50 \mathrm{Mbps}$ (13\%).

\begin{tabular}{|c|c|c|c|}
\hline Arquivos & & & \\
\hline \multirow{5}{*}{$\begin{array}{l}\text { Distribuição por } \\
\text { região }\end{array}$} & Centro-Oeste & 11 & \\
\hline & Nordeste & 14 & \\
\hline & Norte & 6 & \\
\hline & Sudeste & 47 & \\
\hline & Sul & 21 & \\
\hline \multirow{5}{*}{ Natureza jurídica } & Público federal & 25 & \\
\hline & Público estadual & 23 & \\
\hline & Público municipal & 32 & \\
\hline & Privado com fins lucrativos & 1 & \\
\hline & Privado sem fins lucrativos & 18 & \\
\hline \multirow{5}{*}{ Porte da instituição } & & Remuneradas & Voluntárias \\
\hline & Nenhuma & 3 & 61 \\
\hline & De 1 a 9 pessoas & 43 & 27 \\
\hline & De 10 a 50 pessoas & 38 & 6 \\
\hline & Mais de 50 pessoas & 12 & 1 \\
\hline
\end{tabular}




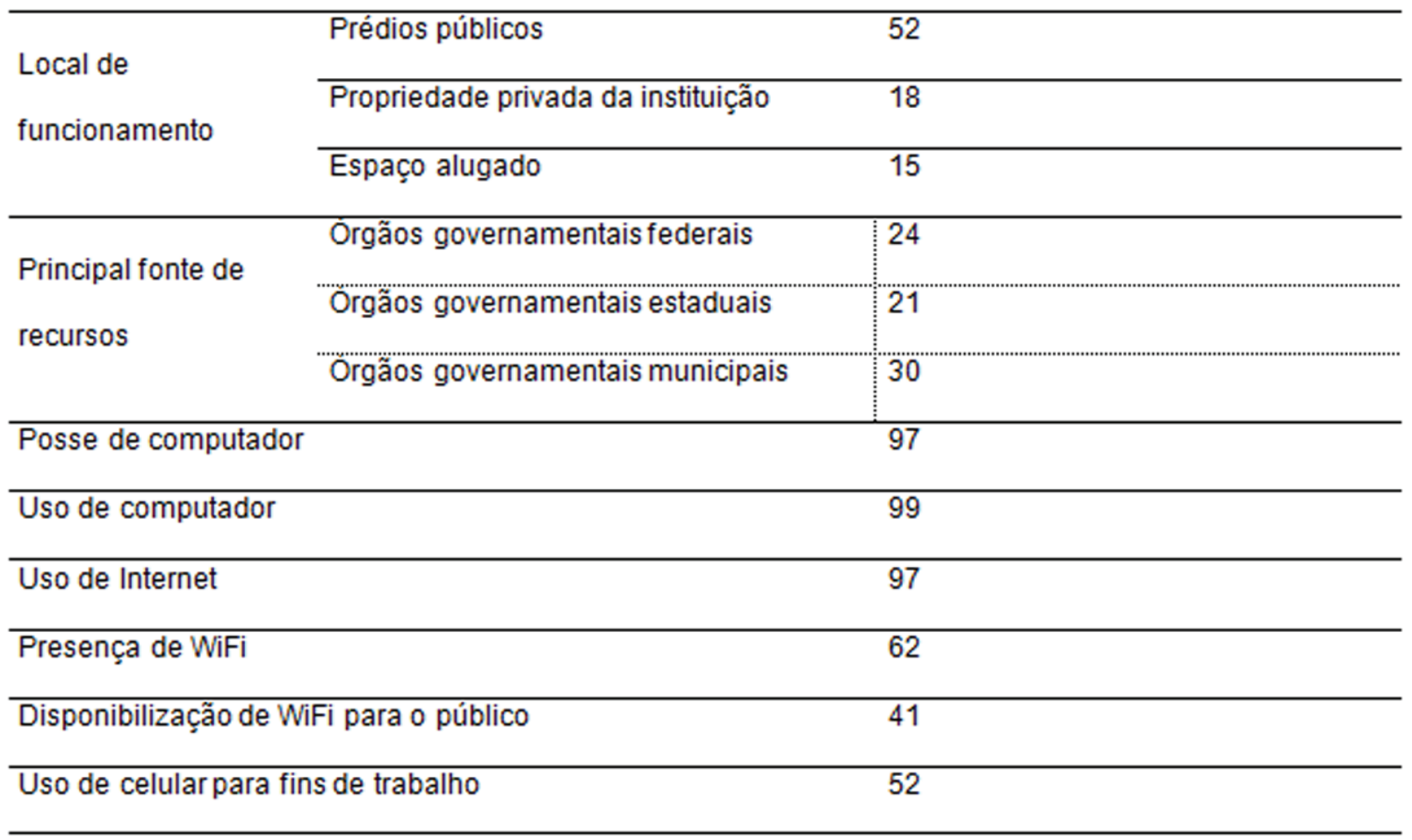

Tabela 1 - Proporção de arquivos, por indicadores de perfil e infraestrutura de TIC.

\section{Uso das TIC na digitalização de acervos}

A digitalização de acervos consiste em procedimento de conversão de materiais para formatos digitais que tem por objetivo, além de garantir a preservação, promover a democratização do acesso aos bens culturais e a difusão do conhecimento, com a disponibilização dos conteúdos digitalizados para o público. Em instituições de guarda e produção cultural, a promoção dessa iniciativa implica em estudo minucioso do acervo existente, seleção das obras a serem digitalizadas, estudo técnico específico acerca do procedimento a ser aplicado, recursos financeiros e tempo de planejamento e execução, bem como conhecimento do público e avaliação do uso pretendido para o conteúdo digitalizado (Silva, 2005).

Diante desse quadro, os equipamentos culturais brasileiros enfrentam de- safios impostos à organização institucional, à gestão de recursos e ao tratamento documental para a digitalização dos acervos. A pesquisa TIC Cultura busca levantar informações sobre o panorama atual dos equipamentos culturais em relação à presença de acervos, implantação de processos de digitalização e disponibilização dos bens culturais em novos formatos para os públicos.

Em 2016, a posse de acervos era uma característica comum entre todos os tipos de equipamentos, abrangendo quase a totalidade de instituições entre arquivos $(98 \%)$, museus $(98 \%)$, bibliotecas $(97 \%)$, bens tombados (94\%) e pontos de cultura $(94 \%)$, justamente os que focalizam guarda e memória. Ainda assim, somente entre arquivos ${ }^{i v}$, museus e pontos de cultura o processode digitalização dos acervos já estava ocorrendo na maioria das instituições (Gráfico 1). 
Total de equipamentos culturais (\%)

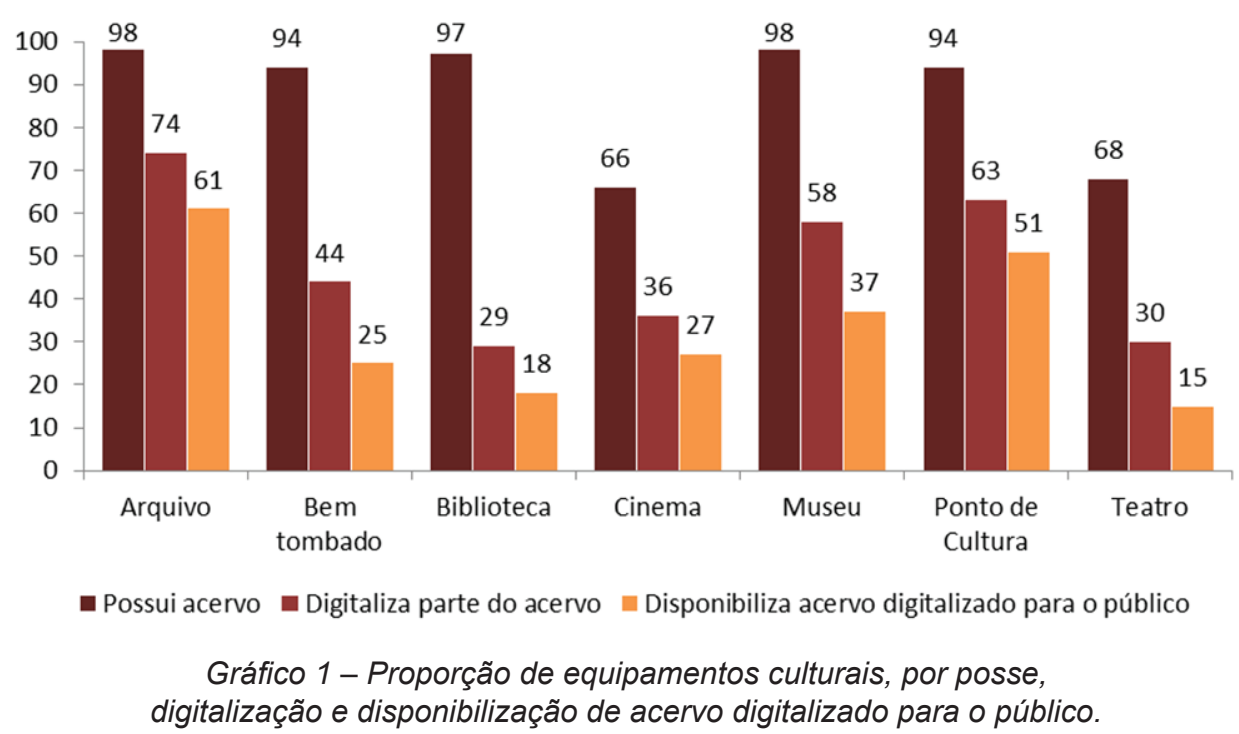

Os arquivos destacam-se no que se refere à digitalização dos acervos dada a natureza de sua atividade e à maior presença e uso das TIC, sendo estes, em conjunto com os cinemas, os equipamentos com os maiores percentuais de posse e uso de computador e de Internet.

Apesar da alta digitalização de acervos entre os arquivos, é possível observar diferenças entre as diversas regiões do país, apontando para disparidades regionais na apropriação dessas ferramentas (Gráfico 2). A região Centro-Oeste teve destaque com $85 \%$ dos arquivos que digitalizam acervo, seguida da região Sul com $80 \%$. A diferença entre a região Norte $(27 \%)$ e as demais regiões é significativa, porém, pela margem de erro, não se pode afirmar que há diferenças entre as demais regiões.

Total de equipamentos culturais (\%)

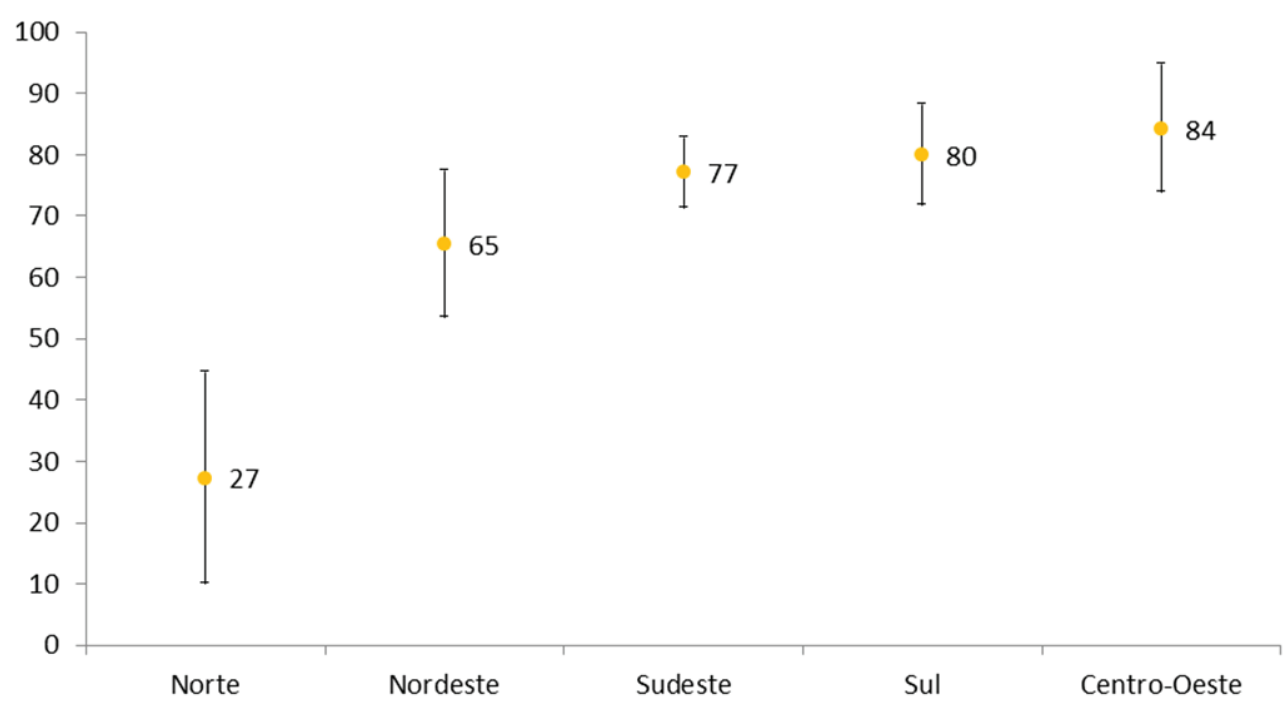

Gráfico 2 - Proporção de arquivos que digitalizam acervo, por região. 
Ainda que a posse de acervos fosse quase generalizada, os tipos de materiais que compunham as coleções apresentaram variações conforme o perfil e escopo de atuação das instituições. Em relação aos tipos de acervos existentes, os arquivos apresentaram maior presença de documentos históricos ou registros arquivísticos (91\%), manuscritos ou documentos originais (85\%), livros, revistas ou jornais $(83 \%)$ e fotografias, cartazes, mapas ou partituras (82\%). Com exceção da categoria de livros, revistas ou jornais, a maioria das instituições estava digitalizando ou já havia digitalizado esses tipos de acervos (Gráfico 3).

Total de equipamentos culturais (\%)

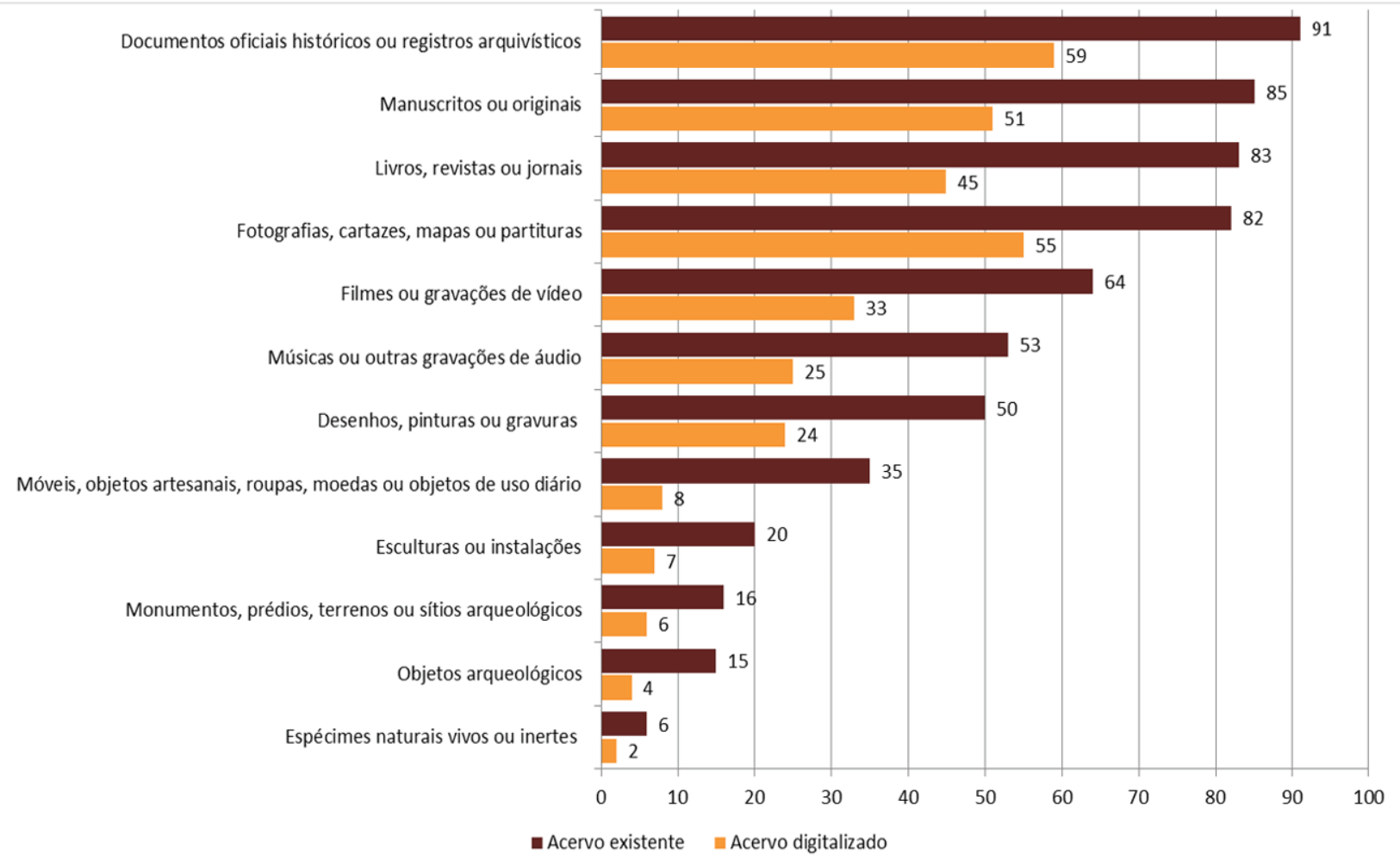

Gráfico 3 - Proporção de arquivos, por tipo de acervo existente e digitalizado.

A maior parte das instituições, no entanto, havia digitalizado menos da metade dos itens de seus acervos (Gráfico 4). Nesse aspecto, é preciso considerar que os percentuais variam tanto em relação à presença dos diferentes tipos de acervos nas instituições, como também dados os diversos tipos de materiais, que implicam em diferentes processos de digitalização. Ainda assim, trata-se de um processo ainda incipiente, mesmo entre as instituições que tem como objetivo a guarda e preservação de acervos, como é o caso dos arquivos. 
Total de equipamentos culturais (\%)

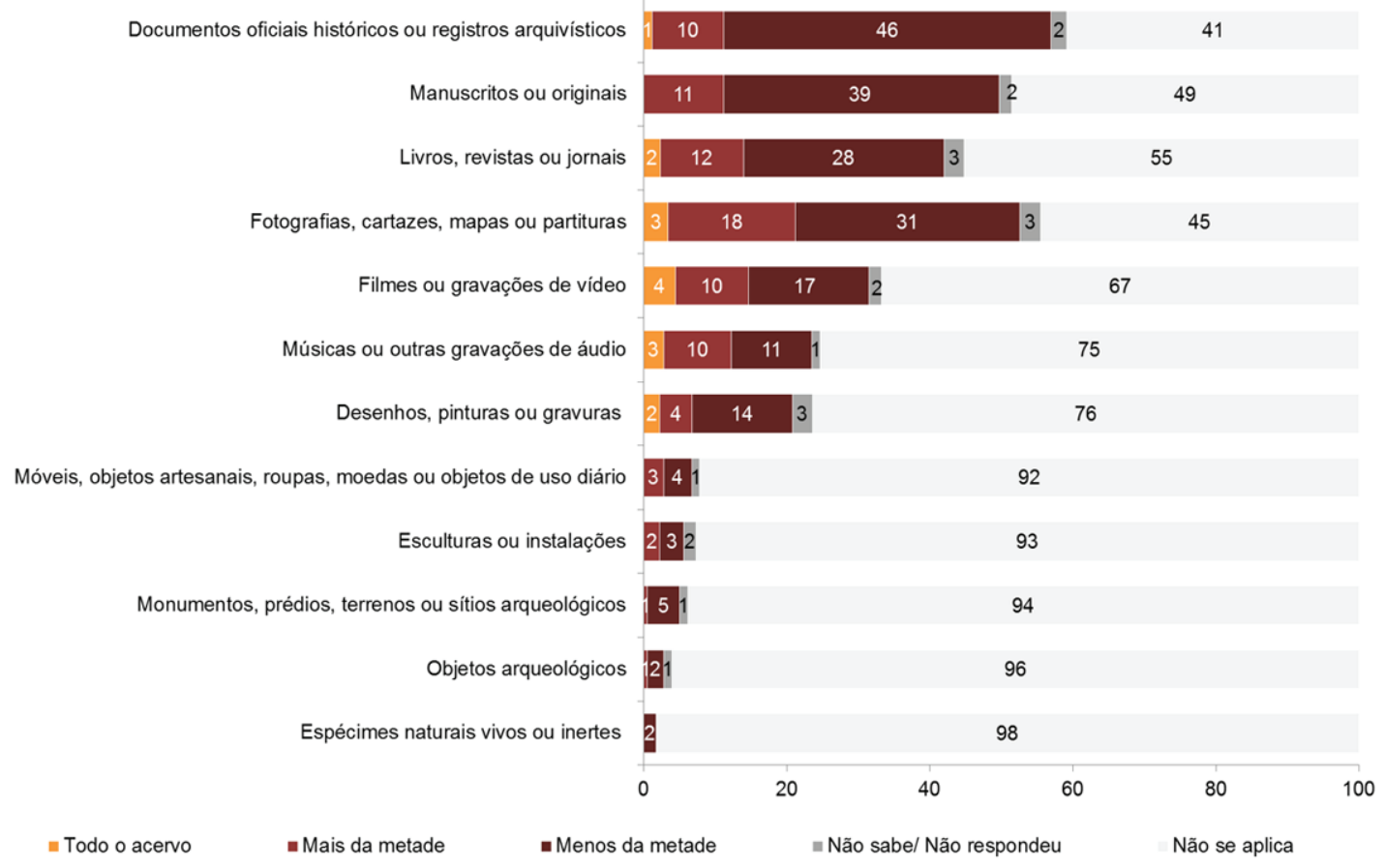

Gráfico 4 - Proporção de arquivos, por quantidade de acervo digitalizado.

As dificuldades para digitalização de acervos mencionadas correspondiam à falta de financiamento $(79 \%)$,seguida da falta de equipe qualificada $(60 \%)$. En- tre os arquivos, como principal dificuldade mencionada destacou-se a falta de financiamento, citada por $60 \%$ das instituições (Gráfico 5).

Total de equipamentos culturais (\%)

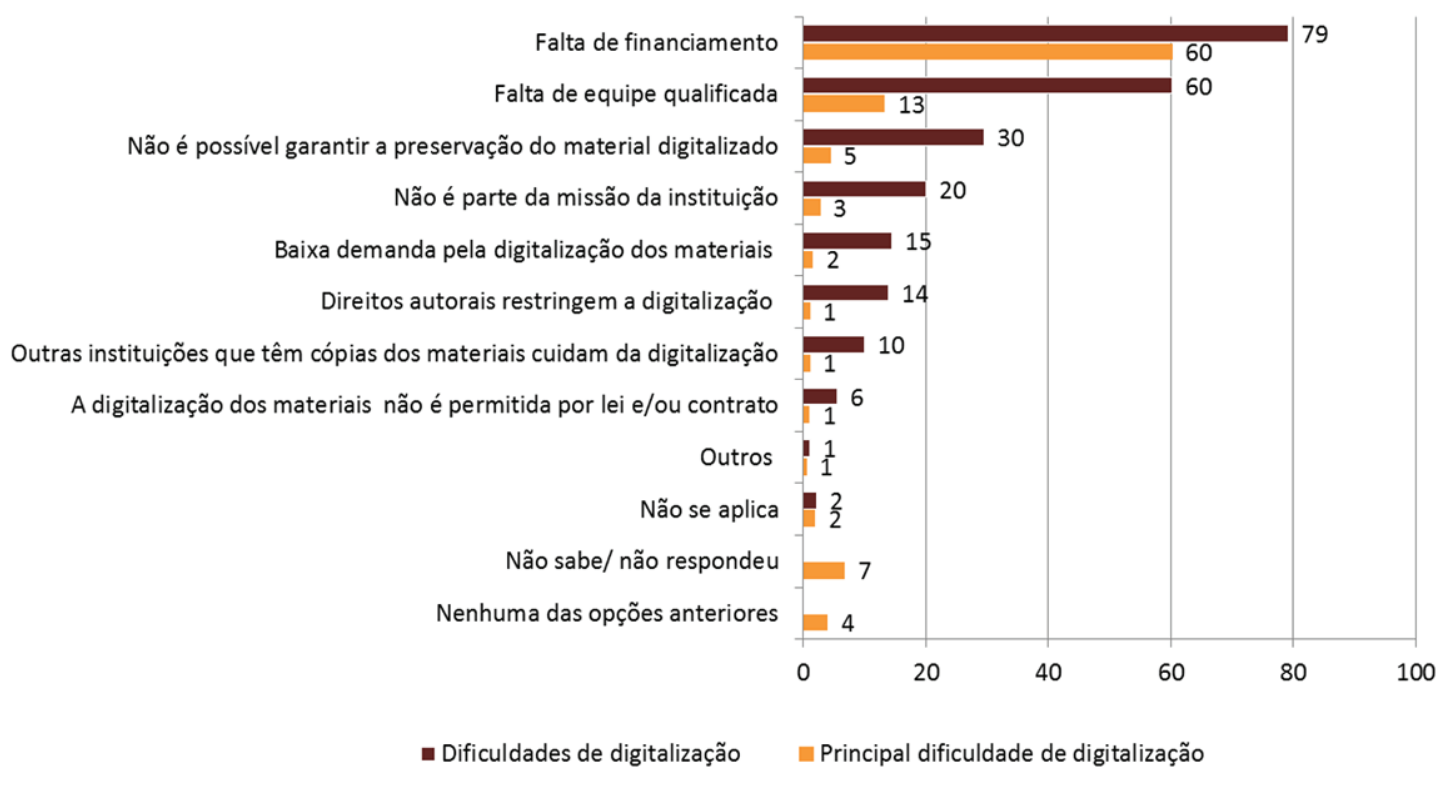

Gráfico 5 - Proporção de arquivos, por dificuldades de digitalização. 
Cabe notar que as dificuldades para digitalização relacionadas às restrições colocadas por direitos autorais foram mencionadas por apenas $14 \%$ dos arquivos. Aqui, é preciso considerar que a restrição colocada pelos direitos autorais está relacionada sobretudo ao uso que é feito do acervo digitalizado, em especial no que se refere à distribuição da cópia. Nesse sentido, a ausência de tais restrições dentre as principais dificuldades para a digitalização de acervos pode ser entendida também por sua baixa disponibilização.

Isso porque, ainda que a disponibilização do acervo digitalizado para o públi- co ocorresse em mais da metade dos arquivos (61\%) (Gráfico 1), de modo geral a disponibilização desses materiais em formato digital não ocorria em websites das instituições, websites de terceiros, perfis ou páginas em redes sociais. $\mathrm{O}$ acesso era majoritariamente ofertado nos locais de funcionamento das próprias instituições, sendo estes mencionados por $59 \%$ dos arquivos. Já a disponibilização em pelo menos uma plataforma digital (como Facebook, YouTube, Instagram, Wordpress ou Blogspot ou WhatsApp) ocorreu em $40 \%$ dos arquivos e a disponibilização nos websites das instituições foi mencionada por apenas $30 \%$ dos arquivos (Gráfico 6).

Total de equipamentos culturais (\%)

Total de equipamentos culturais (\%)

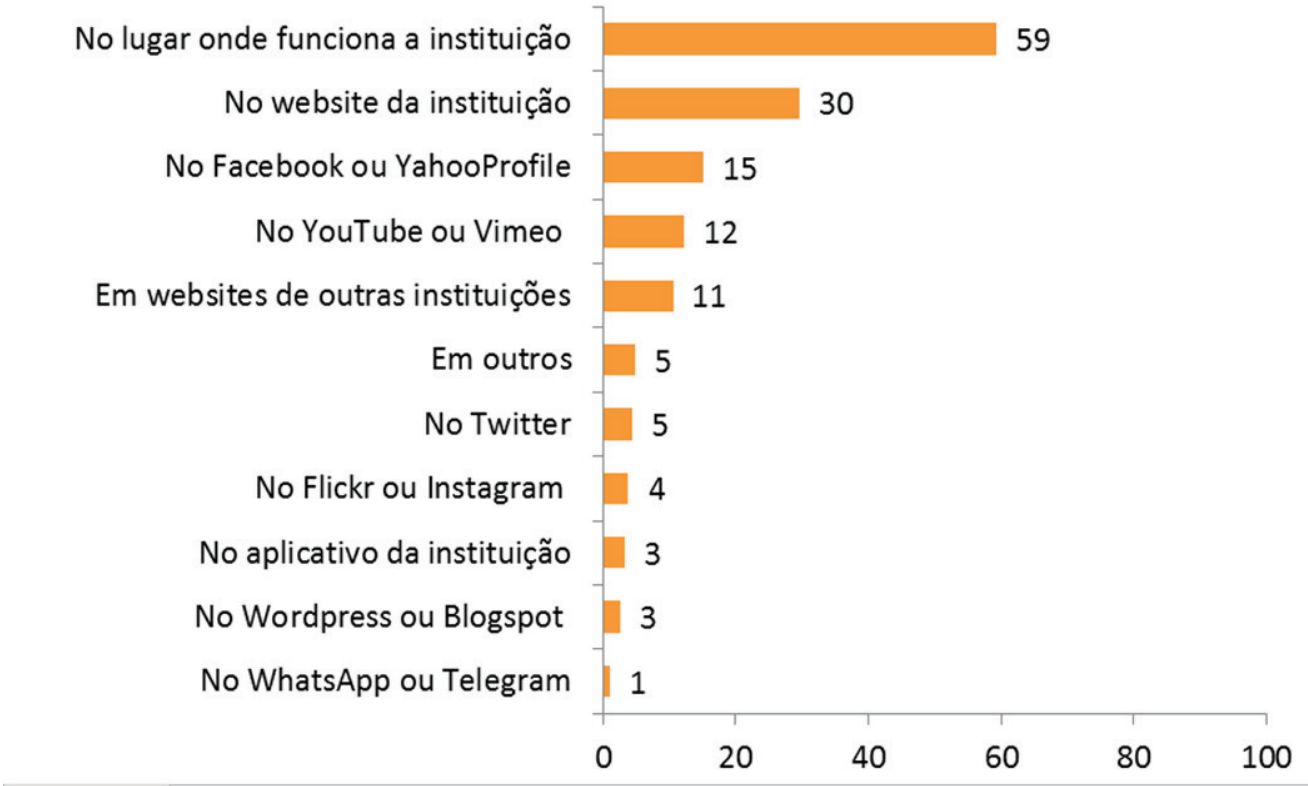

Gráfico 6 - Proporção de arquivos, por forma de disponibilização do acervo digitalizado.

Tais indicadores revelam que, mesmo nos casos em que o acervo foi digitalizado, sua disponibilização na Internet foi menor do que seu acesso ofertado de forma presencial. Assim, a digitalização de acervos, além de incipiente, parece estar mais voltada à preservação dos materiais que à sua difusão. Aqui, novamente, os dados mostram um contexto em que as TIC poderiam ampliar formas de acesso aos bens culturais, com possibilidades que ainda não estão sendo plenamente aproveitadas entre os arquivos brasileiros. 


\section{Considerações finais: Agenda para políticas públicas}

A pesquisa TIC Cultura investiga o uso das tecnologias de informação e comunicação no campo da cultura, tendo como horizonte subsidiar as políticas culturais em âmbito nacional e as agendas internacionais relativas à promoção dos direitos culturais, frente aos desafios postos pela sociedade da informação e do conhecimento.

Devido às poucas estatísticas disponíveis a respeito do setor, o levantamento de indicadores sobre o uso que os equipamentos culturais fazem das TIC é fundamental. Os dados obtidos pela investigação visam contribuir, em especial, para a formulação de políticas públicas específicas da área, de forma a gerar insumos para gestores públicos, pesquisadores, profissionais da cultura, instituições culturais, academia e sociedade civil.

No que diz respeito especificamente aos arquivos brasileiros, os indicadores mostram que a infraestrutura de TIC está bastante presente nessas instituições, sobretudo se comparada a outros tipos de equipamentos culturais (CGI.br, 2017b). O uso dessas tecnologias para a digitalização de acervos, no entanto, ainda que seja realizada pela maior parte das instituições e se destaque também na comparação com outros tipos de equipamentos culturais, é incipiente. A maior parte dos arquivos havia digitalizado menos da metade dos itens de seus acervos, sendo a falta de financiamento e de qualificação das equipes as principais barreiras apontadas para a digitalização. Mesmo entre aqueles que possuíam acervo digitalizado, a maior parte o disponibilizava para o público na própria instituição e não de forma on-line, parecendo indicar que a digitalização está mais voltada à preservação que à difusão desses materiais.

Assim, o aprimoramento do uso das TIC entre os arquivos brasileiros é de funda- mental importância para o maior desenvolvimento do processo de digitalização e disponibilização dos acervos, revelando um potencial ainda pouco explorado dessas ferramentas para ampliação do acesso à cultura.

Para alterar esse cenário, as políticas públicas precisam ter foco no investimento financeiro e na capacitação de recursos humanos para a digitalização, de modo que tais instituições possam ampliar a oferta de bens e serviços culturais pela Internet, disponibilizando-os de forma mais ampla e acessível para a população.

\section{Referências}

COMITÊ GESTOR DA INTERNET NO BRASIL-CGI. br. Cultura e tecnologias no Brasil: Um estudo sobre as práticas culturais da população e o uso das tecnologias de informação e comunicação. São Paulo: CGI.br, 2017a. Disponível em:<http://cetic.br/publicacao/cultura-e-tecnologias-no-brasil/>.Acesso em 20 set. 2017.

COMITÊ GESTOR DA INTERNET NO BRASIL - CGI.br. Pesquisa sobre o uso das tecnologias de informação e comunicação nos equipamentos culturais brasileiros: TIC Cultura 2016. São Paulo: CGI.br, 2017b. Disponível em <http://cetic.br/publicacao/pesquisa-sobre-o-uso-das-tecnologias-da-informacao-e-comunicacao-nos-equipamentos-culturais-brasileiros/>.Acesso em 17 jan. 2018.

CONSELHO NACIONAL DE ARQUIVOS - Conarq. (n.d.). Câmara Técnica de Documentos Eletrônicos. Disponível em: <http://www.conarq. arquivonacional.gov.br/index.php/documentos-eletronicos-ctde>. Acesso em 10 nov. 2017.

EUROPEAN COMISSION. The DigiCULT Report: Technological landscapes for tomorrow's cultural economy: Unlocking the value of cultural heritage. Luxembourg: Office for Official Publications of the European Communities, 2002.

INSTITUTO BRASILEIRO DE GEOGRAFIAE ESTATÍSTICA - IBGE. Perfil dos estados e dos municípios brasileiros: Cultura 2014. Rio de Janeiro: IBGE, 2015. Disponível em: <http://biblioteca.ibge.gov.br/visualizacao/livros/liv95013.pdf>. Acesso em 08 nov. 2017. 
Lei n. 8.159, de 8 de janeiro de 1991. Dispõe sobre a política nacional de arquivos públicos e privados e dá outras providências. Disponível em: <http:// www.planalto.gov.br/ccivil_03/leis/L8159.htm>. Acesso em 20 jul. 2017.

MINISTÉRIO DA CULTURA - Minc. As metas do Plano Nacional de Cultura. São Paulo: Instituto Via Pública; Brasília: Minc, 2012. Disponível em: <http://pnc.cultura.gov.br/2013/01/01/2533/>. Acesso em 20 set. 2017.

ORGANIZAÇÃO DAS NAÇÕES UNIDAS PARA A EDUCAÇÃO,ACIÊNCIAEACULTURA-UNESCO. Institute for Statistics. The 2009 Unesco framework for cultural statistics. Montreal: UNESCO Institute for Statistics, 2009. Disponível em: <http://unesdoc.unesco.org/images/0019/001910/191061e. pdf>. Acesso em 20 jul. 2017.

ORGANIZAÇÃO DAS NAÇÕES UNIDAS PARA A EDUCAÇÃO, A CIÊNCIA E A CULTURA - UNESCO. Towards knowledge societies. Paris: Unesco Publishing, 2005.

ORGANIZAÇÃO DAS NAÇÕES UNIDAS PARA A EDUCAÇÃO, A CIÊNCIA E A CULTURA - UNESCO. Conference of Parties to the Convention on the Protection and Promotion of the Diversity of Cultural Expressions, Sixth ordinary session. Item 11 of the provisional agenda: Draft operational guidelines on the implementation of the Convention in the digital environment. Paris, UNESCO Headquarters, Room II, 12-15 June 2017.

UNIÃO INTERNACIONAL DE TELECOMUNICAÇÕES - UIT. Partnership on Measuring ICT for Development. Final WSIS Targets Review: Achievements, challenges and the way forward. Genebra: ITU, 2014. Disponível em: <https://www.itu.int/en/ITU-D/ Statistics/Documents/publications/wsisreview2014/ WSIS2014_review.pdf>. Acesso em: 20 set. 2017.

SILVA, R. R. G. Manual de digitalização de acervos: Textos, mapas e imagens fixas. Salvador: Edufba, 2005. Disponível em: <https://repositorio.ufba.br/ri/ bitstream/ufba/141/4/Manual\%20de\%20digitalizacao\%20de\%20acervos.pdf>. Acesso em 20 set. 2017.
I Luciana Piazzon Barbosa Lima. Mestre em Estudos Culturais pela Escola de Artes, Ciências e Humanidades da Universidade de São Paulo (EACH-USP). coordena a pesquisa TIC Cultura no Centro Regional de Estudos para o Desenvolvimento da Sociedade da Informação (Cetic.br). São Paulo, Brasil. Contato: luciana@nic.br

II Isabela Bertolini Coelho. Mestre em Estatística pelo Instituto de Matemática e Estatística da Universidade de São Paulo (IME-USP) Estatística no Centro Regional de Estudos para o Desenvolvimento da Sociedade da Informação (Cetic.br). São Paulo, Brasil. Contato: isabela@nic.br

III A pesquisa completa apresenta também indicadores sobre a presença das instituições na Internet por meio de websites e redes sociais e sobre as atividades realizadas na rede, incluindo ações de comunicação, gestão, governo eletrônico, relacionamento com o público e recursos oferecidos em plataformas digitais. Além desses, também traz informações sobre a gestão de TI nessas instituições e a percepção das contribuições e barreiras trazidas pelo uso de computador e Internet. Para acesso a todos os indicadores, ver: http://cetic.br/ pesquisa/cultura/indicadores

IV Nos arquivos brasileiros, o estímulo à gestão de documentos digitais teve amparo institucional através da Câmara Técnica de Documentos Eletrônicos, um grupo de trabalho multidisciplinar que teve por objetivo "definir e apresentar ao Conselho Nacional de Arquivos normas, diretrizes, procedimentos técnicos e instrumentos legais sobre gestão arquivística e preservação dos documentos digitais, em conformidade com os padrões nacionais e internacionais"(Conselho Nacional de Arquivos [Conarq], n.d., para. 2). 